\title{
HIV-induced neuroinflammation: impact of PAR1 and PAR2 processing by Furin
}

\author{
Vatsal Sachan ${ }^{1} \cdot$ Robert Lodge $^{2} \cdot$ Koichiro Mihara $^{3,4} \cdot$ Josée Hamelin $^{1} \cdot$ Christopher Power $^{5} \cdot$ Benjamin B. Gelman ${ }^{6}$.

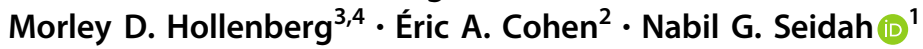

Received: 25 July 2018 / Revised: 12 December 2018 / Accepted: 17 December 2018 / Published online: 25 January 2019

(c) ADMC Associazione Differenziamento e Morte Cellulare 2019

\begin{abstract}
HIV-associated neurocognitive disorders (HAND) is a syndrome defined by neurocognitive deficits that are driven by viral neurotoxins, cytokines, free radicals, and proteases expressed in the brain. This neurological disease has also been linked to activation of Protease-Activated Receptors 1 and 2 (PAR1,2). These receptors are highly expressed in the central nervous system and are upregulated in HAND. Secretory basic-amino-acid-specific Proprotein Convertases (PCs), which cleave precursor proteins at basic residues, are also induced in HAND. They are vital for many biological processes including HIV-1 entry into cells. The cytoprotective role of Furin, PC5, and PACE4 has been linked to the presence of a potential PC-cleavage site $\mathrm{R}_{41} \mathrm{XXXXR}_{46} \downarrow$ in PAR1. Furthermore, Furin binds PAR1 and both are trapped in the trans-Golgi-network (TGN) as inactive proteins, likely due to the intermediary trafficking role of phospho-Furin acidic cluster sorting protein 1 (PACS1). Nothing is known about PAR2 and its possible recognition by PCs at its putative $\mathrm{R}_{31} \mathrm{XXXXR}_{36} \downarrow$ processing site. The present study implicates PACS1 in the retrograde trafficking of PAR1 to the TGN and demonstrates that the cytosolic extreme Cterminal tail of PAR1 contains an acidic phosphorylatable PACS1-sensitive domain. We further show the requirement of $\mathrm{Asn}_{47}$ in PAR1 for its Furin-dependent TGN localization. Our data revealed that Furin is the only convertase that efficiently cleaves PAR2 at $\operatorname{Arg}_{36} \downarrow$. N-glycosylation of PAR2 at $A_{30}$ reduces the efficacy, but enhances selectivity of the Furin cleavage. Finally, in co-cultures comprised of human neuroblastoma SK-N-SH cells (stably expressing PAR1/2 and/or Furin) and HIV-1-infected primary macrophages, we demonstrate that the expression of Furin enhances neuronal cell viability in the context of PAR1- or PAR2-induced neuronal cytotoxicity. The present study provides insights into early stages of HIV-1 induced neuronal injury and the protective role of Furin in neurons co-expressing PAR1 and/or PAR2, as observed in HAND.
\end{abstract}

Edited by R.A. Knight

Supplementary information The online version of this article (https:// doi.org/10.1038/s41418-018-0264-7) contains supplementary material, which is available to authorized users.

Nabil G. Seidah

seidahn@ircm.qc.ca

1 Laboratory of Biochemical Neuroendocrinology, Montreal Clinical Research Institute (affiliated to the University of Montreal), 110 Pine Ave West, Montreal, QC H2W1R7, Canada

2 Laboratory of Human Retrovirology, Montreal Clinical Research Institute (affiliated to the University of Montreal), 110 Pine Ave West, Montreal, QC H2W1R7, Canada

3 Inflammation Research Network-Snyder Institute for Chronic

\section{Introduction}

The main neurological consequence currently associated with HIV-1 infection in the central nervous system (CNS) is HIV-associated neurocognitive disorders (HAND) [1-4]. It is a spectrum syndrome with a prevalence of $>25 \%$ among HIV-infected populations worldwide despite increased availability of antiretroviral therapy (ART) [5]. The most

Diseases, Department of Physiology and Pharmacology, Faculty of Medicine, University of Calgary, Calgary, AB, Canada

4 Department of Medicine, Faculty of Medicine, University of Calgary, Calgary, AB T2N4N1, Canada

5 Department of Medicine, University of Alberta, Edmonton, AB T6G2S2, Canada

6 Department of Pathology, University of Texas Medical Branch Houston, Galveston 77555 TX, USA 
common neurocognitive deficits include: memory loss, reduced concentration and decision-making abilities, disorientation, and psychomotor slowing. The most advanced stage of HAND, termed HIV-associated dementia is linked to neuroinflammation, neuronal injury, and death [6]. Shortly after primary infection, HIV-infected macrophages and possibly lymphocytes cross the blood-brain-barrier and infect glial cells, especially microglia and perhaps astrocytes. These events lead to the release of several HIV-1 proteins, free radicals, proteases, and proinflammatory cytokines in the microenvironment of the CNS, contributing to sustained inflammation in the brain. This chronic inflammation also drives the dysregulation and activation of numerous proteins in the CNS, including the proteaseactivated receptors (PARs) $[7,8]$ and the proprotein convertases $(\mathrm{PCs})[9,10]$.

The PAR family comprises four cell-surface localized 7 transmembrane receptors (PAR1, PAR2, PAR3, and PAR4) belonging to the G-Protein-Coupled-Receptor (GPCR) superfamily [11]. Unlike other GPCRs, which are activated upon binding soluble ligands, PARs are activated when their extracellular N-terminal segment is cleaved by a protease, e.g., thrombin for PAR1 (Fig. 1A), thereby uncovering a tethered ligand at the newly formed $\mathrm{N}$-terminus [11]. This ligand then binds the second extracellular loop of the receptor leading to a conformational change in the transmembrane helices of the protein, eventually triggering downstream signaling (Fig. 1B) [11-13]. The levels of cellsurface PAR1 are regulated by protease-induced internalization. It is well established that PAR1 is primarily cleaved by thrombin at its N-terminal $\operatorname{Arg}_{41} \downarrow$ and PAR2 by trypsin at $\operatorname{Arg}_{36} \downarrow$ [14]. Once cleaved, PAR1 and PAR2 can trigger pro- or anti-inflammatory signaling pathways, depending on the cognate protease and microenvironment. The processed receptors are internalized and sent to lysosomes for degradation via clathrin/dynamin-dependent pathways. Activated PAR1 and PAR2 have been linked to neuroinflammation and neuronal death $[15,16]$. PAR1 activation leads to the proliferation of microglia resulting in a sustained release of inflammatory cytokines in the brain [17]. PAR2 activation was shown to be neurotoxic in rat hippocampus [18].

The proprotein convertases (PCs), a family of nine secretory proteases that exhibit multiple functions in various tissues including the CNS [9], could play a significant role in regulating PAR-associated signaling. Indeed, the basicamino-acid-specific Furin, PC5A, PACE4, and PC7 are upregulated in the CNS by inflammation [10]. These PCs cleave their substrates either in the trans-Golgi network (TGN) or cell-surface/endosomes at the consensus motif (Arg/Lys) $2 \mathrm{Xn}(\operatorname{Arg}) \downarrow$ (where "Xn" corresponds to $0,1,2$, or 3 spacer amino acids, and the arrow denotes the P1 cleavage site) $[9,19]$.

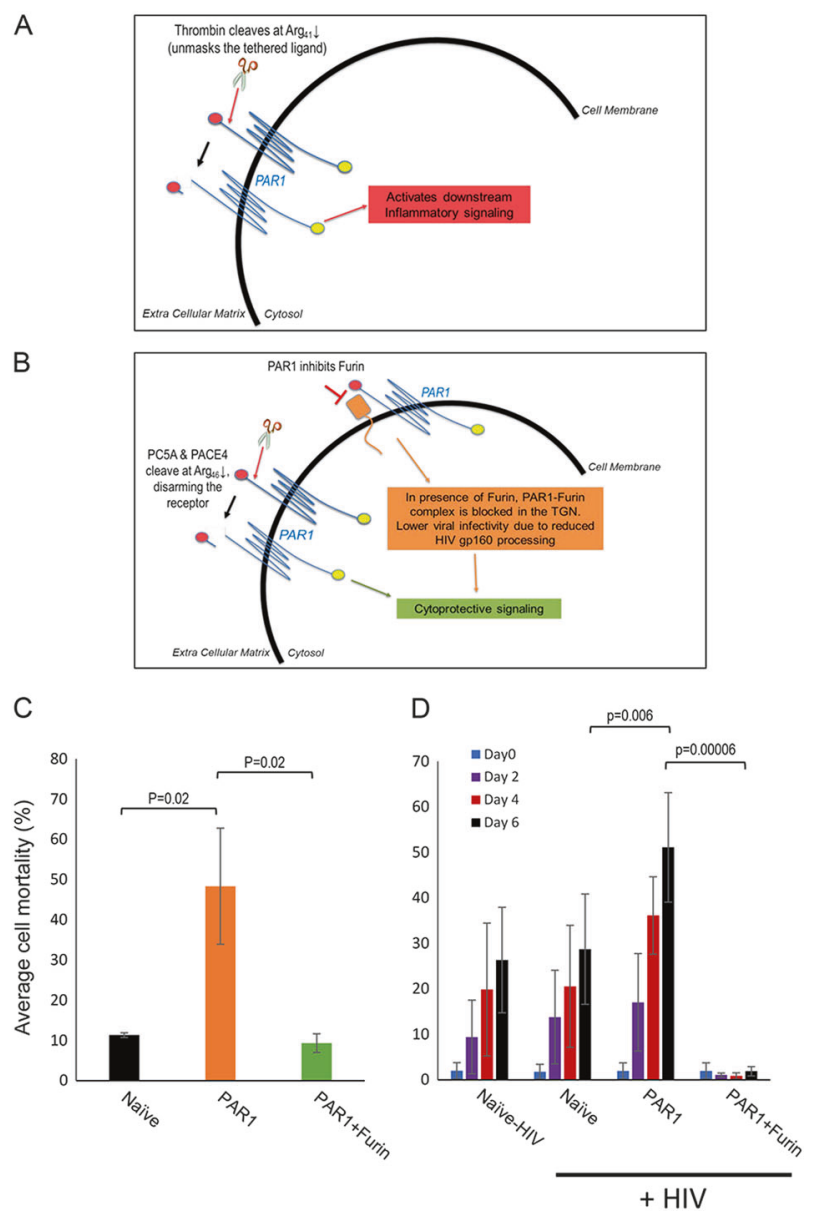

Fig. 1 Role of PAR1 in HIV-1 associated neurocognitive disorders. A Thrombin (red) cleaves PAR1 at $\operatorname{Arg}_{41} \downarrow$ and activates a cascade of inflammatory signaling. B Schematic representation of the cytoprotective role of Furin, PC5A, and PACE4 in PAR1-induced inflammation. PC5A and PACE4 (green) cleave downstream to the thrombin cleavage site, i.e., at $\operatorname{Arg}_{46} \downarrow$ and render the receptor inactive. Furin (orange) traps the receptor in the TGN, possibly in an inactive form. The red and yellow circles denote mcherry and eYFP tags on PAR1, respectively. C Cell viability of SK-N-SH naive, PAR1, and PAR1/Furin stably expressing cells was measured using an MTS assay. Mortality was measured after $48 \mathrm{~h}$ by analyzing and comparing the fraction of living cells in thrombin-untreated and thrombin-treated cells (\% cell mortality). PAR1 expressing cells show high mortality rate in presence of thrombin. However, Furin overexpression abolishes this effect. Thrombin treatment: $10 \mathrm{nM}$ (experiment performed in triplicate). D Cell viability was measured using FACS analysis (see materials and methods) at different time points post-co-culture with HIV-1 infected macrophages. PAR1 expressing cells show high mortality. However, Furin has a protective effect by trapping PAR1 in the TGN. $N=5$ (macrophage from five different donors); Bars represent mean $\pm \mathrm{SD}$, and $p$ was determined using a one-tailed student $T$-test. Values of $p<0.05$ are considered significant and are shown for each condition. Within PAR1 expressing cells the increment of cell mortality is significantly higher at all time points. Note that at all time points the presence of Furin significantly reduces cell mortality in presence of PAR1

We previously reported the upregulation of inflammation-related (e.g., IL-1 $\beta$, TNF $\alpha$ ) transcripts together with those of Furin, PC5, PACE4, PC7, and PAR1 in 
the brains of HAND patients [10]. In accordance with the presence of a potential PC-recognition motif $\operatorname{Arg}_{41}$ XXX$\mathrm{XArg}_{46} \downarrow$ in the extracellular N-terminal segment of PAR1, we demonstrated that PAR1 inhibits membrane-bound Furin, which in turn downregulates functional PAR1 by trapping it in the trans Golgi network (TGN). In addition, the soluble PC5A and PACE4 can disarm cell-surface PAR1 through cleavage at Pro-Arg ${ }_{41}$-Ser-Phe-Leu-Leu$\operatorname{Arg}_{46} \downarrow$ Asn, resulting in a decreased calcium mobilization in response to thrombin stimulation, suggesting that these PCs are cytoprotective [10]. We showed that wild-type (WT) PAR1, but not its R46A mutant, lowers viral infectivity by inhibiting the ability of Furin to process the HIV-1 gp160 into gp120/gp41 [10]. These data suggested a protective [Furin/PC5A/PACE4]-PAR1-interaction pathway that may counterbalance the effects of HAND-associated pathogenesis [10], as schematically summarized in Fig. 1B. However, the participation of PCs in PAR2 processing and regulation of neuronal viability are unknown and are addressed in the present study.

\section{Results}

\section{Inhibition of PAR1 by Furin: consequences on neuronal cell viability}

To assess the effects of Furin on PAR1-regulated neuronal survival (Fig. 1B), we obtained pools of stable transfectants of cDNAs coding for PAR1 or PAR2 in the presence or absence of C-terminally V5-tagged Furin [10]. Human neuroblastoma cells SK-N-SH, often used for studies of HIV-1 neuropathogenesis [20-22] can be differentiated to adopt a neuronal phenotype [23]. Western blot analyses confirmed the high expression of these PARs and Furin in these neuronal stable transfectants (Supplementary Figure S1). Viability analyses by an MTS-tetrazolium assay revealed that these cells exhibited $<5 \%$ mortality over six days of culture (Supplementary Figure S2). Furthermore, inspection of these cells did not reveal any morphological changes compared to naive cells (not shown). We next incubated control naive SK-N-SH cells or their stable transfectants with thrombin $(10 \mathrm{nM})$ for $48 \mathrm{~h}$, and cell viability measured. In the absence of PCs, PAR1 overexpression resulted in $48.4 \pm 14.4 \%$ cell death. However, the presence of Furin together with PAR1 significantly reduced such thrombin-induced toxicity to $9.3 \pm 3.6 \%$ mortality similar to naive cells (Fig. 1C).

To mimic ex vivo the HIV-1 infected macrophageinduced inflammatory microenvironment of neurons seen in HAND patients, we co-cultured for six days the aforementioned engineered SK-N-SH neuronal cells with HIV-1 infected human monocyte-derived primary macrophages
(MDMs) obtained from four different healthy blood donors [24]. These MDMs were infected ex vivo with HIV-1 for at least six days, and co-cultures were then performed by seeding MDMs on cell culture inserts in wells containing SK-N-SH cells. We measured cell viability at days $0,2,4$, and 6 (Fig. 1D). This configuration ensures that the cocultures are done under conditions (semi-permeable membrane) where contacts between infected macrophages and SK-N-SH cells are negligible. We used two control experimental paradigms: naive SK-N-SH cells incubated with uninfected macrophages (naive-HIV) or with macrophages infected with HIV-1 ( + HIV). In both cases $~ 25 \%$ cell death was observed at day 6 , which might be in part attributed to the presence of endogenous PAR1 in these cells [8], and to the secretion of cathepsin B [21] and/or cathepsin G [24] from HIV-1-infected macrophages. Note that since naive-HIV and naive cells co-cultured with HIVinfected macrophages show the same $\%$ of cell death, it was unlikely that SK-N-SH cells were themselves infected during the co-culture. Cells overexpressing PAR1 alone show high mortality, e.g., $51.1 \pm 12.0 \%$ at day 6 . However, cells expressing PAR1 and Furin are protected from such macrophage-induced neurotoxicity (viability $>98 \%$ ). The above data support a protective role of upregulated Furin in PAR1-induced neuroinflammation seen in HAND patients. The higher viability of PAR1-Furin expressing cells compared to naive cells is likely due to an effect of Furin overexpression in the activation of as yet undefined survival factors. One possibility is the Furin activation of transforming growth factor $\beta 1$ [25] likely resulting in neuronal cytoprotection [26].

\section{Trafficking of PAR1: critical roles of phospho-Furin acidic cluster sorting protein 1 (PACS1) and the Furin-recognition motif in PAR1}

We next investigated the mechanism underlying the observed Furin-induced protective role via analysis of its inhibitory effect on the trafficking of PAR1 to the cellsurface and its retention in the TGN [10]. Previous studies showed that the cytosolic protein PACS1 escorts membrane-bound Furin from the cell-surface to the TGN [27] through its interaction with an acidic motif in the cytosolic tail of Furin containing two phosphorylated serine (pS) residues: $\mathrm{pS}_{773} \mathrm{DpS}_{775}[28,29]$. Since PAR1 also contains an acidic phosphorylatable cluster (ESSDPSSYNSSGQLMAS 406 ) in its C-terminal extreme cytosolic tail [30], it was plausible that Furin retention of PAR1 in the TGN or vice versa might involve their interaction with PACS1. Indeed, we have already demonstrated that PACS1 can retain PAR1 in the TGN of HEK293 cells [10] (Supplementary Figure S3). We now further show that in HEK293 cells PACS1 can also retain in the TGN the 
A

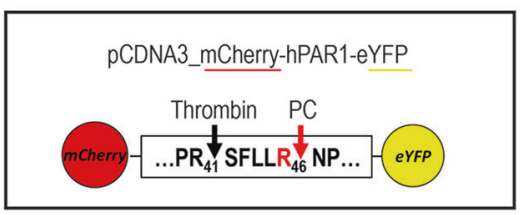

B

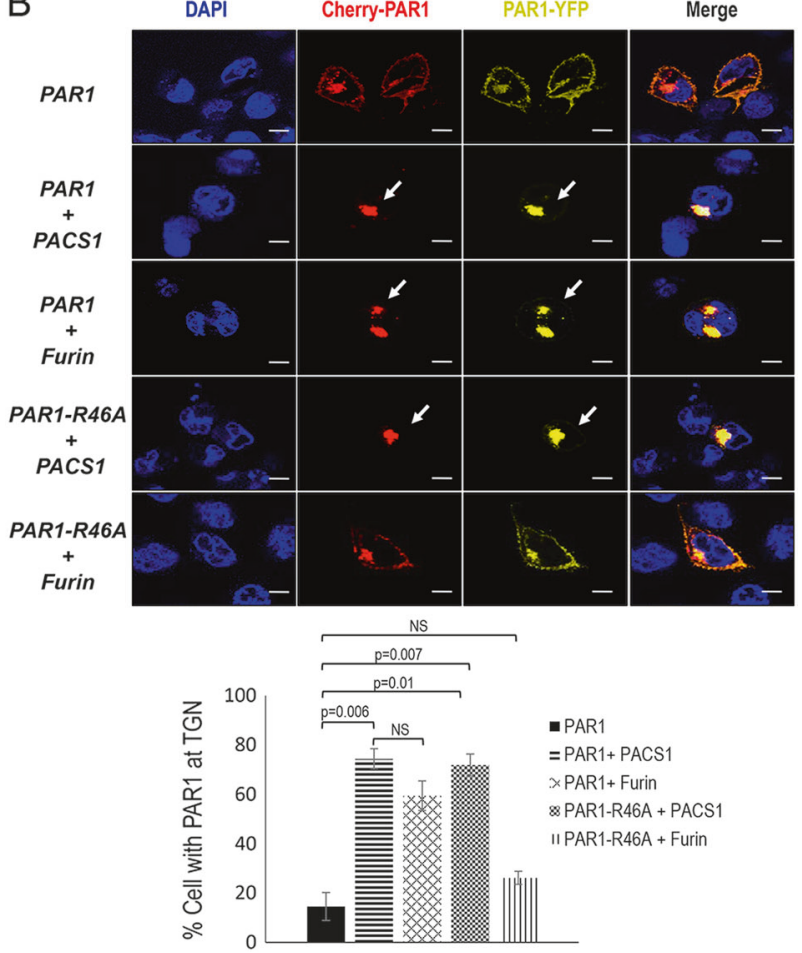

Fig. 2 PACS1 regulates the trafficking of PAR1, independent of Furin. A Schematic representation of mcherry-PAR1-eYFP construct cloned in pcDNA3. The red and yellow circles denote mcherry and eYFP tag on the $\mathrm{N}$-terminus and the $\mathrm{C}$-terminus of PAR1, respectively. The black arrow denotes the thrombin cleavage site at $\operatorname{Arg}_{41} \downarrow$ and the red arrow denotes the downstream PC- cleavage site at $\operatorname{Arg}_{46} \downarrow$. B PAR1 trafficking in Furin-deficient CHO-FD11 cells. The first panel shows PAR1 localized on the cell-surface. When co-expressed with either PACS1 or Furin, PAR1 is trapped in the TGN (white arrow) in panels 2 and 3. PACS1 is able to trap PAR1-R46A mutant (unresponsive to PCs) in the TGN (panel 4). However, Furin is unable to change the subcellular localization of PAR1-R46A as opposed to WT PAR1. The results are from one representative experiment out of three independent ones $(N=3)$. Scale $=20 \mu \mathrm{m}$. The bottom panel presents the quantitation of the images with at least 15 cells/condition analyzed for each of the three experiments and presented as a mean $\%$ of cells with $\mathrm{PAR} 1$ stuck at the TGN $\pm \mathrm{SD}$. Values of $p<0.05$ (determined using a two-tailed $T$-test) are considered significant and are shown for each condition

PAR1-R46A mutant that does not interact with Furin [10] (Supplementary Figure S3; see Golgin97 co-localization), suggesting an additional Furin-independent role of PACS1. To confirm this point, we repeated the co-expression experiment in CHO-FD11 cells that are Furin-deficient [31]. Upon co-expression of Furin or PACS1 with PAR1 it was observed that in this Furin-deficient cell line PAR1 was significantly trapped in the TGN (Fig. 2B). However, while the same result was observed in the co-expression of PACS1 with the PAR1-R46A mutant, the latter was no longer predominantly sorted to the TGN in the presence of only Furin and its localization resembled that of PAR1 alone.

To define the motif in PAR1 that is critical for its interaction with PACS1, we generated a PAR1 construct lacking the above C-terminal acidic-phosphorylatable residues, i.e., PAR1- $\Delta 390-406$ (PAR1- $\Delta$ CT) (Fig. 3A). Like WT PAR1, this deletant translocated to the cellsurface but was no longer sensitive to PACS1, since it did not localize to the TGN upon its co-expression with PACS1. In contrast, Furin can still significantly interact with it and sort it to the TGN (Fig. 3B). We next silenced PACS1 mRNA expression (Supplementary Figure S4), whereupon Furin was no longer able to significantly trap PAR1 in the TGN as compared to a control expressing a scrambled siRNA (Fig. 3C). We conclude that the acidic phosphorylatable C-terminal tail of PAR1 is critical for its interaction with PACS1 and its localization to the TGN, even in absence of Furin. However, in the presence of Furin this domain is dispensable since Furin can interact with PACS1, but also with the PC-like motif $\mathrm{R}_{41} \mathrm{SFLLR}_{46} \mathrm{NP}$ in PAR1 [10].

Notably, PAR1 exhibits an $\mathrm{Asn}_{47}$ at the $\mathrm{P}^{\prime}$ position. It was previously shown that $\mathrm{P}^{\prime}$ Asn prevented Furin cleavage of growth differentiating factor 11 (GDF11) at $\mathrm{RSRR}_{296} \mathrm{NL}$, while Furin cleaved the GDF11-N297D mutant [32]. Accordingly, we generated a N47D mutant of PAR1 and tested for its Furin-induced TGN localization, possible cleavage by PCs and Furin-inhibition of HIV-1 surface glycoprotein gp160 processing. Immunocytochemical data clearly showed that PAR1 and its N47D mutant are not cleaved by Furin, as evident by the red and yellow labeling of PAR1 in the presence of Furin (Fig. 4A). However, in contrast to WT PAR1, Furin can no longer significantly retain PAR1-N47D in the TGN. This unexpected result suggested that either this mutation may result in a partial loss of Furin recognition in the acidic environment of the TGN or its complete loss. Accordingly, we co-expressed the cDNAs of Furin and PAR1 or its N47D mutant together with a cDNA encoding the HIV-1 gp160 Env glycoprotein [10]. The data in Fig. 4B show that both PAR1 and PAR1-N47D inhibit by $20-30 \%$ the Furinprocessing of gp160 into gp120/gp41 (Fig. 4B). This suggests that different from WT PAR1, the mutant PAR1N47D likely binds and inhibits Furin at the cell-surface, but not at the TGN. We conclude that $\mathrm{Asn}_{47}$ is critical for the ability of Furin to retain PAR1 in the acidic environment of the TGN (pH 6.2-6.4), but that PAR1-N47D can still inhibit the proteolytic activity of Furin at the neutral $\mathrm{pH}$ of the cellsurface. 
A

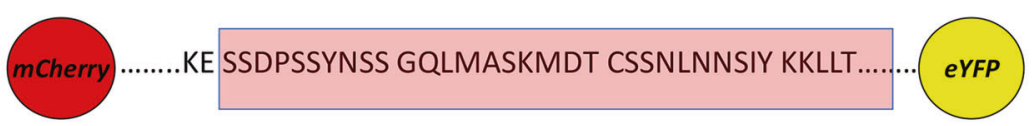

B
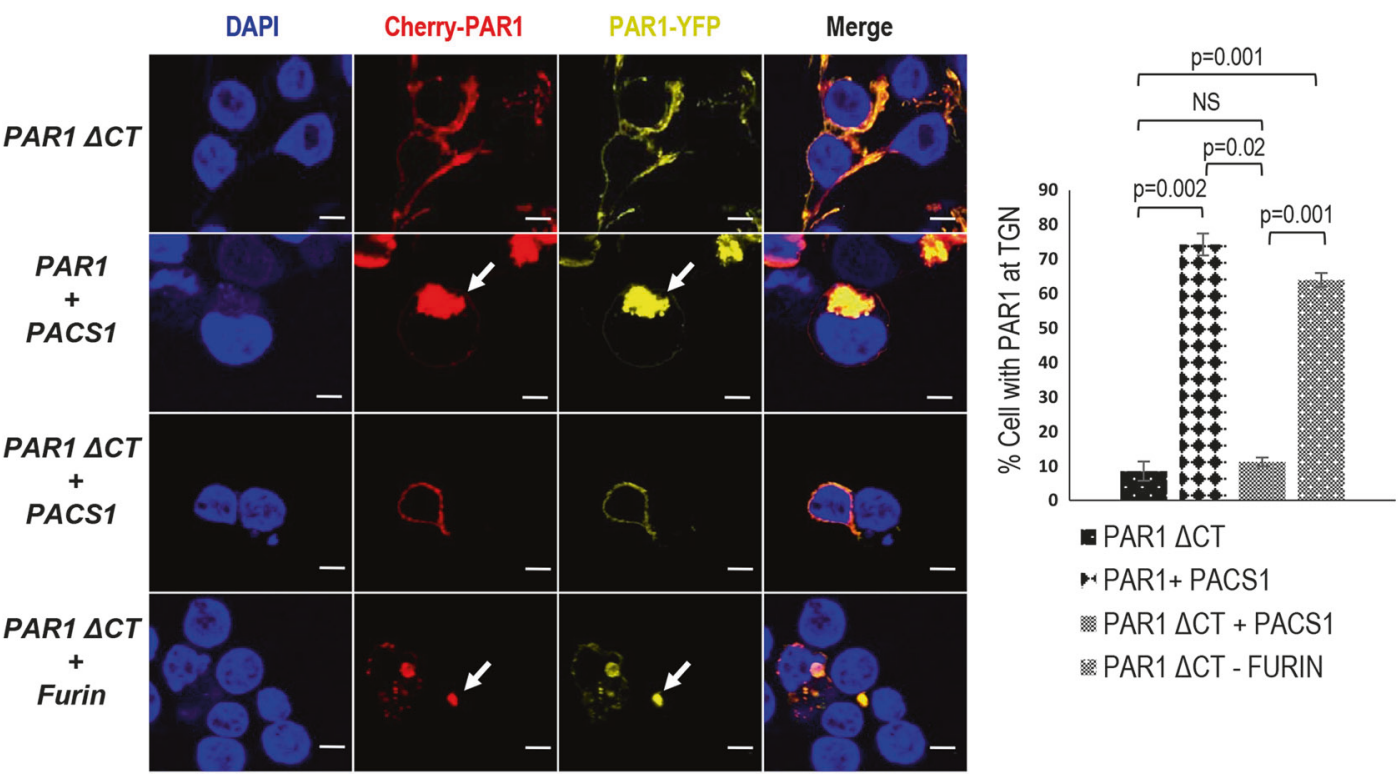

C DAPI Cherry-PAR1 PAR1-YFP Merge
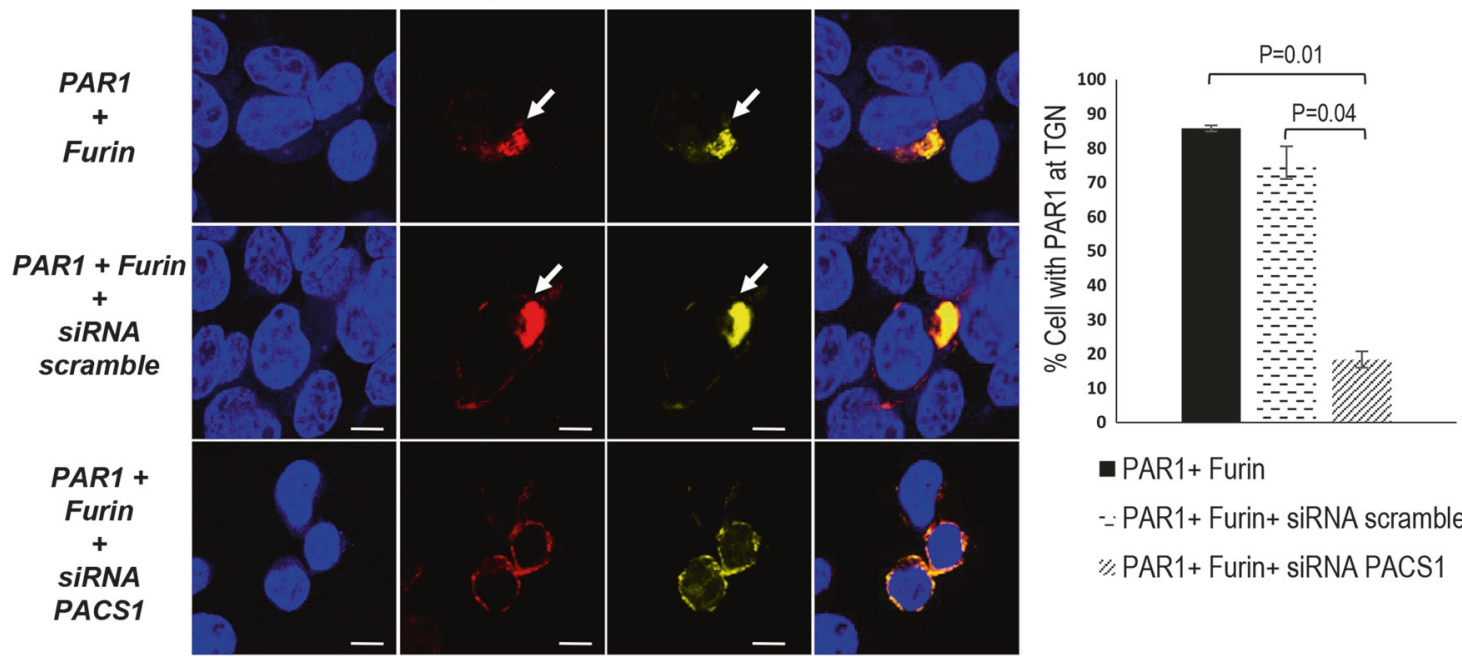

- PAR 1+ Furin

$\because$ PAR1+Furin+ siRNA scramble \% PAR1+ Furin + siRNA PACS1

Fig. 3 Critical role of the cytosolic tail of PAR1 in PACS1-dependent trafficking: knockdown of PACS1 reverses the PAR1 entrapment in the TGN. A Red box shows the deleted portion (amino acids 390-406) of the cytosolic tail of PAR1 rich in Ser residues, some of which are phosphorylated [49]. B PACS1 is unable to trap the PAR1 $\Delta 390-406$ (PAR1 $\Delta$ CT) mutant in the TGN (white arrows) as shown in panel 3, but Furin can, as shown in the last panel. C siRNA knockdown of PACS1 prevents the accumulation of PAR1 in the TGN, which now localizes to the cell-surface, even in the presence of Furin.
PACS1 siRNA ( $25 \mathrm{nM}$ ) was incubated with HEK293 cells transiently transfected with cDNAs coding for PAR1 or PAR1/Furin. A scramble siRNA was used as a control. The results are from one representative experiment out of 2 independent ones $(N=3)$. Scale $=20 \mu \mathrm{m}$. The side panels in $(\mathbf{B}, \mathbf{C})$ present the quantitation of the images with at least 15 cells/condition analyzed for each of the 3 experiments and presented as a mean $\%$ of cells with PAR 1 stuck at the TGN \pm SD. Values of $p<0.05$ (determined using a two-tailed $T$-test) are considered significant and are shown for each condition 


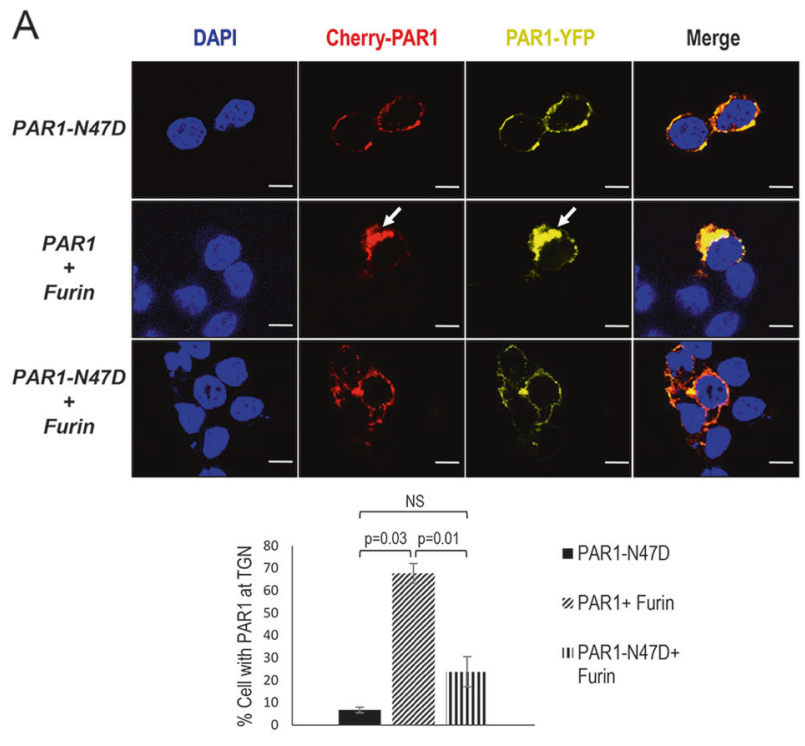

B

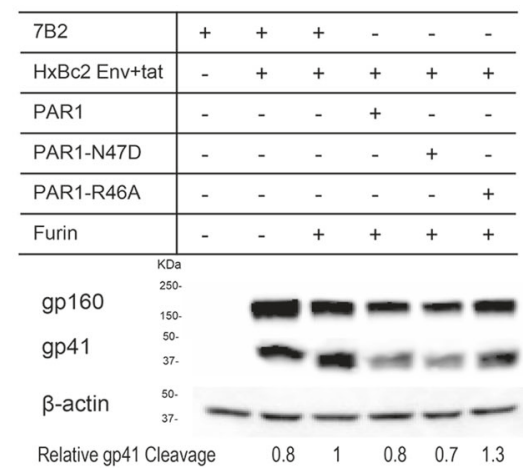

Fig. 4 Role of the highly conserved $\mathrm{Asn}_{47}$ at the P1' position of PAR1. A Localization of PAR1 vs. PAR1-N47D in presence of Furin. The N47D mutant of PAR1 is mostly on the cell-surface (upper panel) similar to WT PAR1 (Fig. 2B). In the presence of Furin, while the WT localizes to the TGN (middle panel) the N47D mutant does not (last panel). The results are from one representative experiment out of 3 independent ones $(N=3)$. Scale $=20 \mu \mathrm{m}$. The bottom panel presents the quantitation of the images with at least 15 cells/condition analyzed for each of the 3 experiments and presented as a mean $\%$ of cells with PAR1 stuck at the TGN \pm SD. Values of $p<0.05$ (determined using a two-tailed $T$-test) are considered significant and are shown for each condition. B PAR1 and its N47D mutant partially inhibit the Furin cleavage of gp160 into gp41 in HEK293 cells (lanes 4 and 5). As controls, we show that a control protein 7B2 (lane 3) $[9,51]$ or PAR1 R46A that is not recognized by Furin (lane 6) have no effect on gp160 processing by Furin. $\beta$-Actin levels are shown for estimation of proteins deposited from the lysates. The results are from one representative experiment out of 3 independent ones $(N=3)$. Cleavage ratios are calculated by dividing the amount of cleaved gp41 by the sum of gp41 and gp160. These ratios are then normalized to Furin cleavage of gp160, taken as 1

\section{Upregulation of PAR2 in HAND and its processing by Furin}

To determine the HIV-dependent regulation of PAR2, brain transcript analysis was performed on HIV negative (HIV-),
HIV positive $(\mathrm{HIV}+)$, patients with HAND and patients with HAND- and HIV-associated encephalitis (HIVE) $(N=$ 10 for each group) [33]. The data show that the mRNA coding for PAR2 is highly upregulated in the brains of patients suffering from HAND ( $\sim$-fold), and more so in those with HIVE ( 6-fold) (Fig. 5A). Immunohistochemical data obtained from frontal cortex of the brain of HAND patients (Supplementary Table S2) revealed that PAR1 and PAR2 expression were chiefly expressed in neurons, and less so in glial cells (Fig. 5B).

To study the role of the PCs in the processing of PAR2, we used a previously reported PAR2 construct with the $\mathrm{N}$ - and Ctermini fused to mRFP (red) and eYFP (yellow), respectively (Fig. 5C) [34]. Just like PAR1 [10], PAR2 is expressed at the cell-surface co-localizing with the low-density lipoprotein receptor (Supplementary Figure S5). PAR2 also exhibits a PCrecognition motif $\mathrm{NR}_{31} \mathrm{SSKGR}_{36} \downarrow$ in its $\mathrm{N}$-terminal segment. Thus, we co-expressed in HEK293 cells the dual-tagged PAR2 along with potential convertases that were previously shown to be upregulated in HAND, namely Furin, PC5A/ PC5B, PACE4, and PC7 [10]. Cleavage at the N-terminus of PAR2 would lead to the loss of red fluorescence, leaving behind a yellow eYFP tagged membrane-bound C-terminal fragment. When expressed alone, full-length doubly-tagged PAR2 localizes to the cell-surface (Fig. 5D). As previously reported [35, 36], a positive control including a $1 \mathrm{~h}$ incubation of cells with $16 \mathrm{nM}$ trypsin results in $\mathrm{N}$ terminal cleavage of PAR2 (seen by the absence of the mRFP tag in the $2^{\text {nd }}$ panel). Treatment with equimolar (trypsin + anti-trypsin) blocks this cleavage, confirming that loss of the mRFP tag was in fact due to trypsin activity.

In contrast to PAR1, co-expression of PAR2 with Furin led to the loss of the N-terminal red tag $\left(4^{\text {th }}\right.$ panel), suggesting that Furin, like trypsin, can cleave the receptor at its $\mathrm{N}$-terminus. That cleavage occurs at $\operatorname{Arg}_{36} \downarrow$ is supported by the resistance of the PAR2-R36 A mutant to Furin $\left(5^{\text {th }}\right.$ panel). It is important to note that unlike PAR1, where PCs cleave downstream to the thrombin cleavage site and hence disarm it [10], Furin cleaves PAR2 at the same site as trypsin, but the other PC-members do not cleave PAR2 (Supplementary Figure S6A,B).

Since Furin can be active in the TGN, cell-surface or endosomes [29], it was imperative to define where cleavage of PAR2 occurs. To probe this question, we treated PAR2 and Furin expressing cells with two pan-PC inhibitors that also inhibit Furin, namely hexa-D-arginine (D6R) and decanoyl-Arg-Val-Lys-Arg-chloromethyl ketone (decRVKR-cmk). D6R is a predominantly cell impermeable inhibitor of PCs, while dec-RVKR-cmk is a potent cellpermeable inhibitor [37, 38]. Treatment of cells with decRVKR-cmk blocks the Furin cleavage of PAR2, but so does D6R (Fig. 6A). This suggests that Furin cleavage of PAR2 occurs mostly at the cell-surface. 


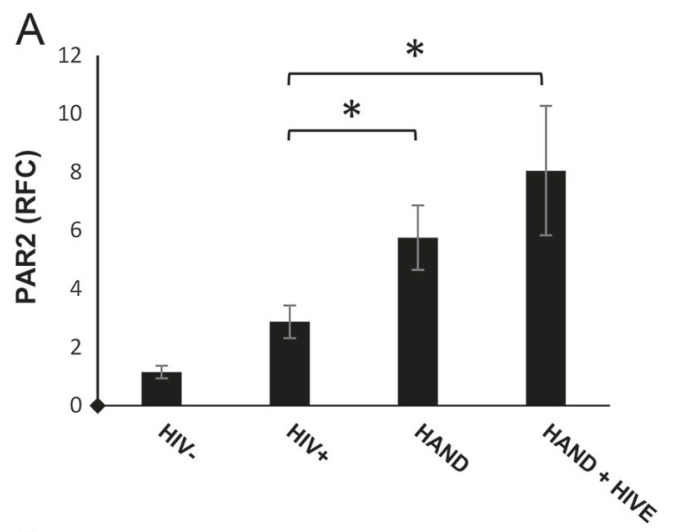

B

C

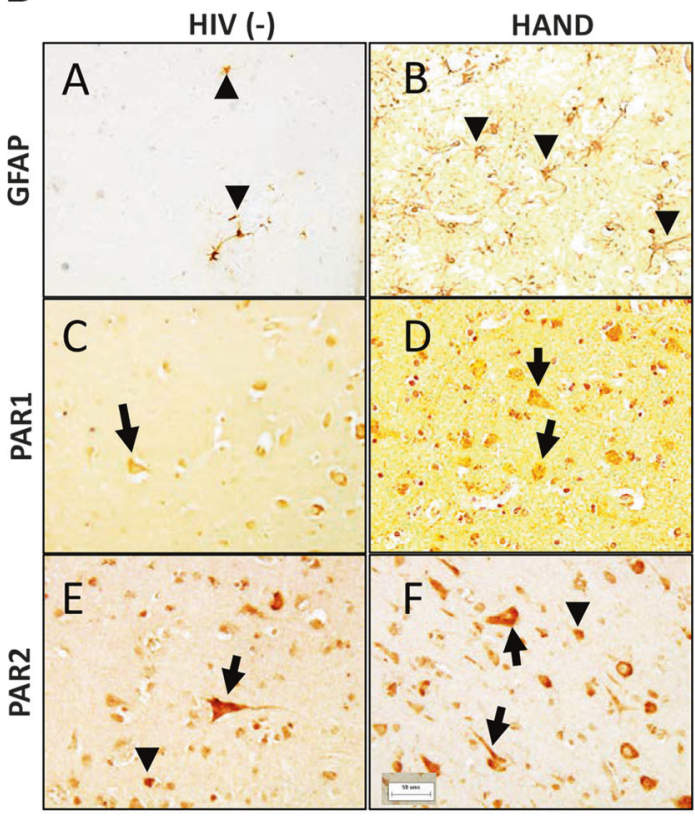

D

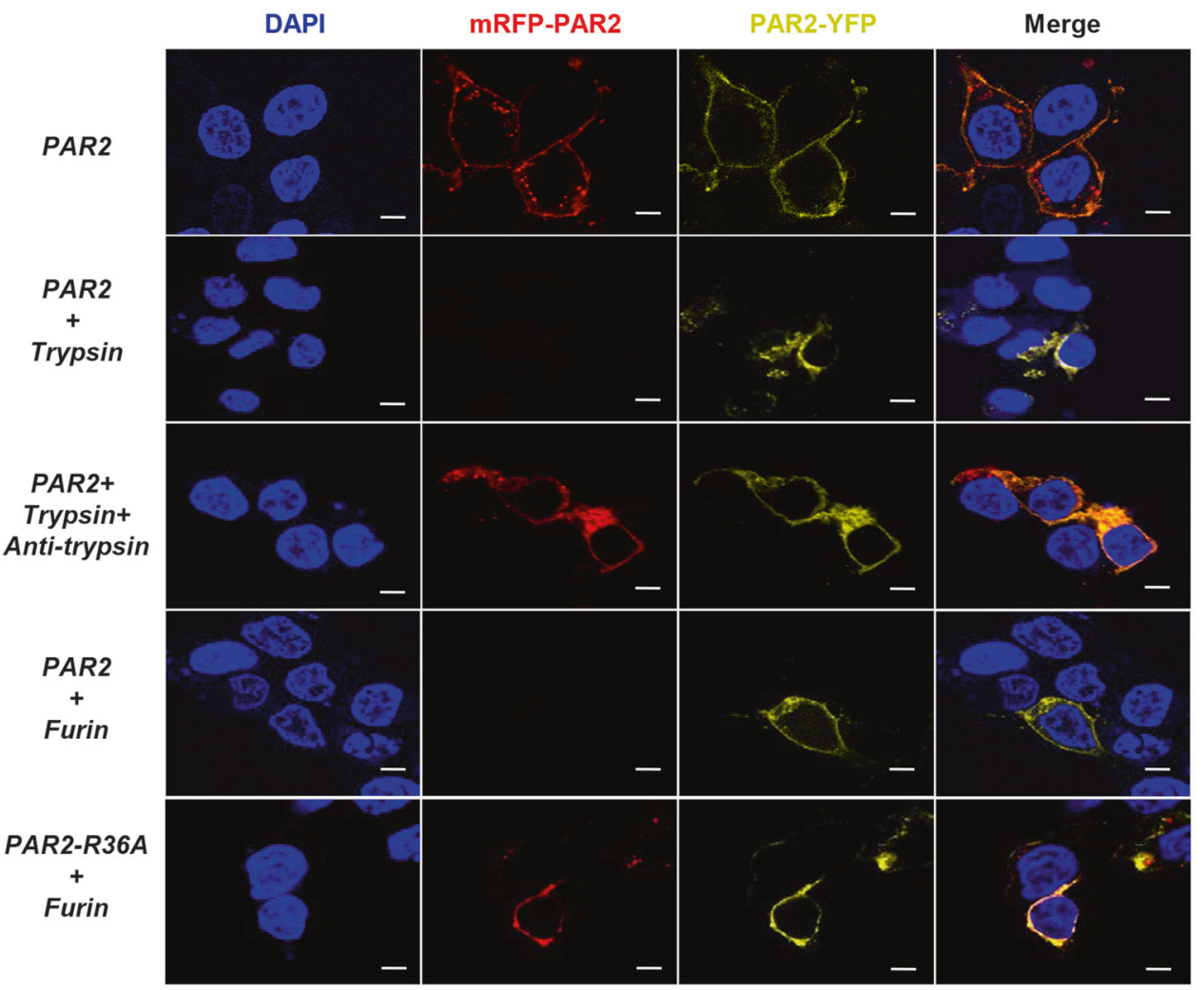

Upon analysis of the PAR2 sequence at Asn-Arg- $\underline{S e r}_{32}$ it was plausible that $\mathrm{N}$-glycosylation at $\mathrm{Asn}_{30}$ that sits in the P7 position of the Furin/trypsin cleavage site (Supplementary Figure S7), could create a steric hindrance and rationalize the high specificity of Furin for PAR2 vs. other PCs. Interestingly, an N30S variant of PAR2 resulting from a single nucleotide polymorphism has been documented (rs616235, minor allele frequency: 0.0345) and reported to enhance PAR2 processing by tryptase and trypsin [39]. Accordingly, we compared in HEK293 cells the processing by PCs of PAR2 to that of PAR2-N30S. The data show that lack of $\mathrm{N}$-glycosylation at $\mathrm{Asn}_{30}$, while enhancing the 
Fig. 5 Upregulation of PAR2 in HAND and its processing by Furin. A PAR2 transcript upregulation in the brains of HAND subjects with HIV-associated encephalitis. Brain transcript analysis was performed on HIV-1 negative (HIV-1-), HIV-1 positive (HIV-1 + ), patients with HAND and patients with HAND and HIV-1 associated encephalitis (HAND + HIVE) ( $N=10$ for each group). The analysis (mean \pm SEM) revealed statistically significant higher transcript levels of PAR2 in the brains of subjects with HAND and HAND + HIVE compared to HIV-1 + patients $(* p<0.05)$. B PAR1 and PAR2 expression in human brain. GFAP $(\mathbf{A}, \mathbf{B})$, PAR1 $(\mathbf{C}, \mathbf{D})$, and PAR2 (E, F) immunoreactivity was present in frontal cortex from HIV (-) (A, $\mathbf{C}, \mathbf{E})$ and HAND $(\mathbf{B}, \mathbf{D}, \mathbf{F})$ patients although all proteins showed increased expression in HAND brains. GFAP, PAR1, and PAR2 were detected in glia (arrowheads) but PAR1 and PAR2 were observed chiefly in neurons (arrows). (Size bar-50 $\mu \mathrm{m})$. C Schematic representation of mRFP-PAR2-eYFP construct cloned in pcDNA3. The red and yellow bubbles denote the mRFP and eYFP tags on the N- and Ctermini of PAR2, respectively. The red arrow denotes the trypsin- and putative PC-cleavage site at $\operatorname{Arg}_{36} \downarrow$. D PAR2 is expressed alone or together with Furin in HEK293 cells. The receptor is cleaved by membrane-bound Furin, as depicted here by the absence of the mRFP fluorescence (panel 4). Trypsin served as a positive control for Furin cleavage, with treatment with anti-trypsin blocking trypsin cleavage (panels 2 and 3, respectively). PAR2-R36A mutant is not cleaved when co-expressed with Furin, as shown in the $5^{\text {th }}$ panel by the presence of both red and yellow fluorescence. The results are from one representative experiment out of 3 independent ones $(N=3)$. Scale $=$ $20 \mu \mathrm{m}$

Furin-cleavage efficacy by $\sim 3$-fold, also results in the $\sim 5$ fold increased ability of PC7 to process PAR2-N30S compared to WT-PAR2, but not the other convertases (Fig. 6B, C). Thus, we conclude that N-glycosylation of PAR2 at $\mathrm{Asn}_{30}$ reduces the processing efficacy of Furin and prevents cleavage by PC7.

\section{Processing of PAR2 by Furin: consequences on neuronal cell viability}

Human monocyte-derived primary macrophages from three independent donors were infected or sham-infected with HIV-1. As for PAR1 (Fig. 1D), these cells were then co-cultured with SK-N-SH cells expressing PAR2 or PAR2/Furin and their viability assessed at co-culture days 0, 2, 4, and 6 (Fig. 7). Control naive cells (lacking PAR2/ Furin \pm HIV-1 infection) exhibited $<7.4 \pm 1.1 \%$ cell death at day 6. Comparatively, we observed at day 6 a significant increase in cell mortality of PAR2-expressing cells $(19.5 \pm 5.1 \%$ translating into an increased average mortality for the three donors of $\sim 2.3$-fold), which was attenuated by $\sim 50 \%$ at days 4 and 6 in cells co-expressing PAR2 and Furin, reaching the levels of naive cells seen in absence of PAR2/Furin (cell mortality $7.8 \pm 0.3 \%$ ). We conclude that as for PAR1 (Fig. 1C), Furin is protective in PAR2-induced neurotoxicity associated with HIV-1 infection.

\section{Discussion}

This is the first report that shows that the proinflammatory PAR2 is a Furin substrate and that the latter is neuroprotective under conditions mimicking neuroinflammation induced by HIV-1 infection that results in the upregulation of PAR1 and PAR2 together with Furin.

HIV-1 infection of the nervous system occurs at seroconversion and is defined by infection of innate immune (myeloid) cells within the nervous system [40]. HAND pathogenesis is widely assumed to be predicated on virusinfected and -activated myeloid cells (microglia, trafficking macrophages) releasing cytotoxic viral proteins and innate immune molecules (e.g., cytokines, proteases) in the CNS $[10,41]$, causing selective neurotoxicity in the frontalstriatal regions of the brain [40].

Activation of PARs via proteases, including PAR1,2, have been linked to inflammation and neuronal death [15, 16]. PAR1 and PAR2 are expressed in hippocampus and amygdala, while PAR2 is also found in thalamus, cortex, and striatum $[42,43]$. Additionally, proteases that signal via PARs are also present in the CNS, e.g., thrombin and its activator Factor X are expressed in neural cell cultures [44, 45]. Trypsin, the primary activator of PAR 2 , is absent from the CNS. However, CNS mast cell tryptase is a potential protease for neuronal PAR2 activation [46, 47].

The roles of the basic-amino-acid-specific PCs in neuroinflammation are still obscure. Recently, we showed that via PAR1 inhibition of Furin and their blockade in the TGN or through PAR1 disarming by PC5A or PACE4 these convertases might act as protective anti-inflammatory proteases (Fig. 1A). In mice, Furin in myeloid cells was also reported to be anti-inflammatory via its ability to activate TGF- $\beta 1$ and its influence on the reduced activity of ADAM17 and hence levels of the proinflammatory cytokine TNF $\alpha$ [48]. Previously, it was demonstrated that PAR2induction upon neuroinflammation prevents neuronal death in mouse brains and that PAR2-deficiency exacerbates HIV1-Tat-induced neuropathological and neurobehavioral deficits [7]. In the present report we demonstrate that Furin is the only PC-like convertase that can process PAR2 (Fig. 5), suggesting that specific cleavage of PAR2 at $\operatorname{Arg}_{36} \downarrow$ by Furin would be neuroprotective. Indeed, co-culture experiments confirmed this conclusion and showed that while PAR2 enhances neuronal death, co-expression of Furin greatly attenuates this phenomenon (Fig. 7), as was also the case for PAR1 (Fig. 1C). It is thus probable that HIV-1infected macrophages secrete factors or proteases, e.g., cathepsins B [21] or cathepsin G [24], which could transform PAR2 into a proinflammatory protein, but that the coexpression of PAR2 and Furin would protect against the cytotoxic effect of such a factor(s), likely via cell-surface 
Fig. 6 Cell-surface cleavage of PAR2 and effect of $\mathrm{Asn}_{30} \mathrm{~N}$ glycosylation on PAR2 processing. A Treating PAR2Furin expressing cells with D6R and dec-RVKR-cmk blocks Furin cleavage, as seen in the two lower panels. The concentration of inhibitors used: $10 \mu \mathrm{M}$ D6R and $25 \mu \mathrm{M}$ decRVKR-cmk. This confirms that PAR2 cleavage occurs mostly at the cell-surface. B Coexpression of PAR2-N30S with all the PCs in HEK293 cells. Note that the N30S mutant is processed more efficiently by Furin and partially by PC7, but not by PC5A and PACE4. The positive control consisted of incubation of PAR2-expressing cells with $12 \mathrm{nM}$ trypsin for 45 min, resulting in the secretion of $\mathrm{a} \sim 30 \mathrm{kDa} \mathrm{N}$-terminal fragment, also seen with Furin and PC7, but not PC5A or PACE4. C Comparison of PAR2 and PAR2-N30S processing by Furin and PC7. Removal of a putative steric hindrance by $\mathrm{N}$ glycosylation at $\mathrm{Asn}_{30}$ makes the $\mathrm{N}$-terminus of PAR2 more accessible to Furin- and PC7cleavage. $\beta$-Actin levels are shown for estimation of proteins deposited from the lysates. The results are from one representative experiment out of 3 independent ones $(N=3)$ except for $(\mathbf{C})$ where $N=2$. Cleavage ratios are calculated by dividing the amount of released $\mathrm{N}$-terminal fragment by the sum of $\mathrm{N}$-terminal fragment and uncleaved PAR2. These ratios are then normalized to Furin cleavage of WT PAR2 and $\beta$ Actin levels, taken as 1
A

PAR2

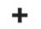

Furin

PAR2

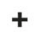

Furin

$+$

DGR

PAR2

$+$

Furin

$+$

dec-RVKR-cmk

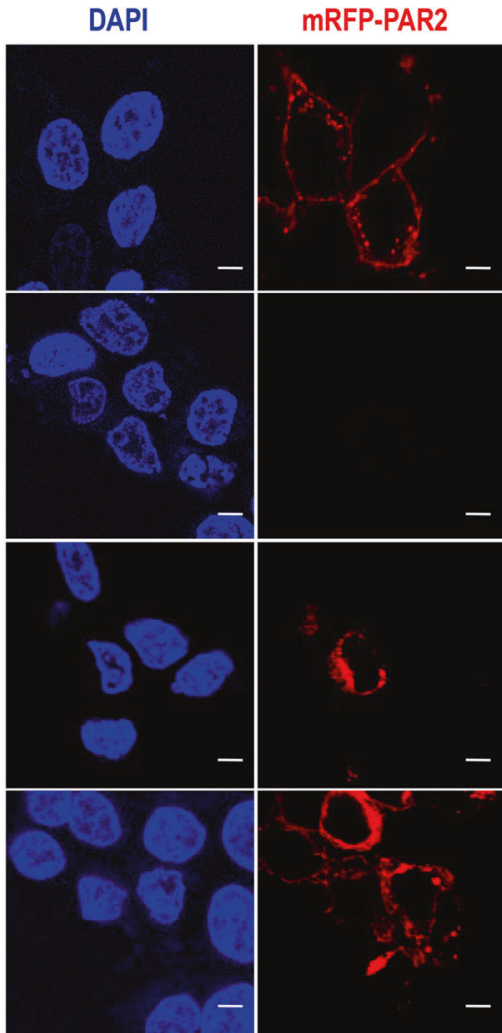

PAR2-YFP

Merge

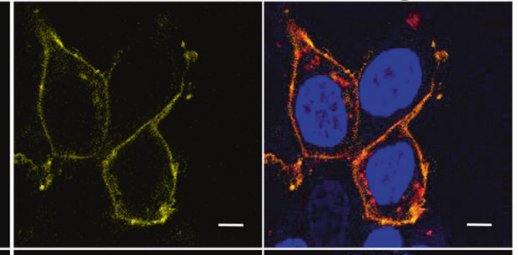

B

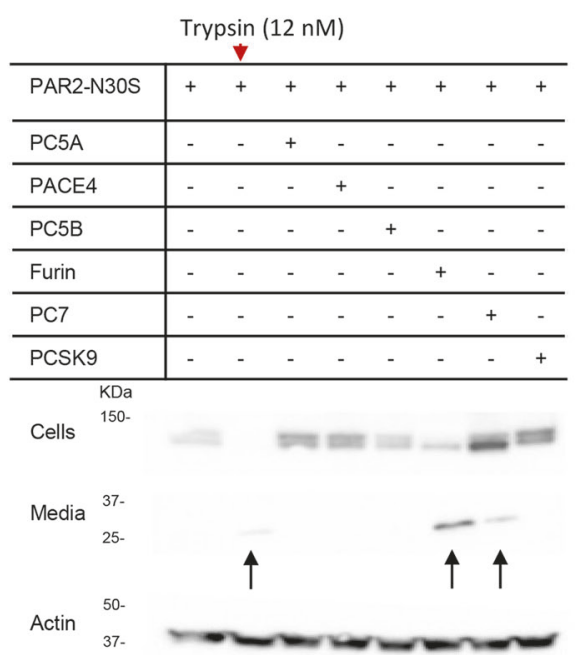

C

\begin{tabular}{|c|c|c|c|c|c|c|}
\hline PAR2 & + & + & + & - & & - \\
\hline PAR2-N30S & - & - & - & + & & + \\
\hline Furin & - & + & - & - & & - \\
\hline PC7 & - & - & + & - & & + \\
\hline 7B2 & + & - & - & + & & - \\
\hline
\end{tabular}

$37-$

25-

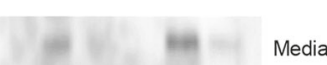

$50-$

37

$\begin{array}{llllll}\text { Relative cleavage extent } & 1 & 0.2 & 0.6 & 3 & 1\end{array}$

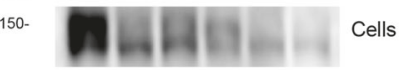

cleavage of PAR2 (inhibited by D6R, Fig. 6) before its interaction with the putative factor secreted by HIV-1 activated macrophages. It is also possible that the Furinmediated cleavage of PAR2 induces a neuroprotective signaling that alleviates the effect of the putative soluble factor (s) secreted by HIV-infected macrophages. Alternatively, cell-surface Furin may also inactivate this soluble factor(s) directly, a subject that would require extensive future studies.
We previously showed that the soluble PC5A and PACE4 disarm PAR1 by cleaving it downstream $\left(\operatorname{Arg}_{46} \downarrow\right)$ from the thrombin cleavage site $\left(\operatorname{Arg}_{41} \downarrow\right)$ and thus protecting from inflammation. The membrane-bound Furin, PC5B, and PC7 do not cleave the receptor, but PAR1, in turn, inhibits the activity of these proteases, especially Furin. In the presence of Furin, PAR1 has also been shown to be trapped in the TGN, likely in a non-functional form [10]. Our present results suggest that PACS1 is directly involved 


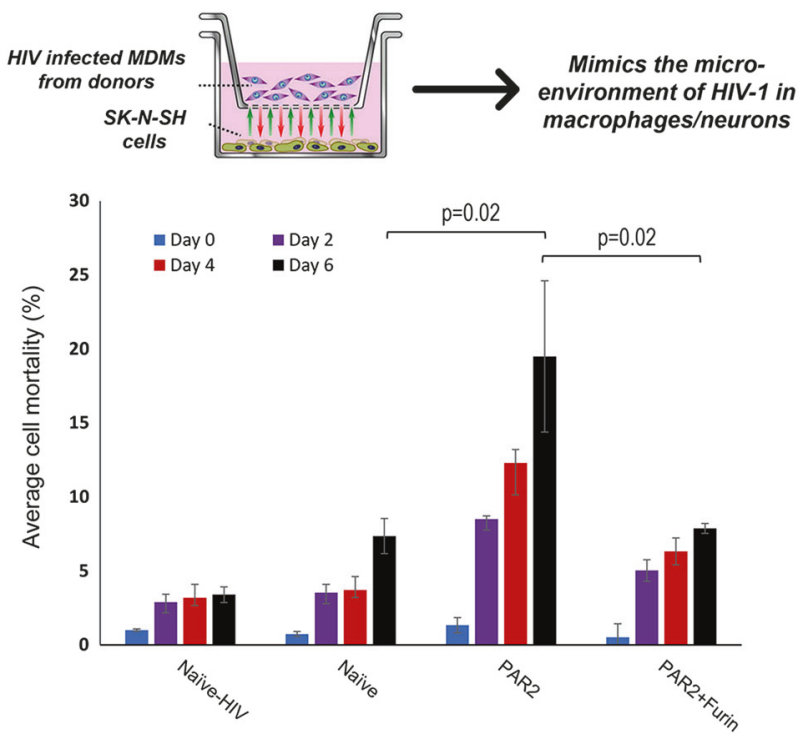

Fig. 7 Furin protects neuronal cells from PAR2-induced toxicity when co-cultured with HIV-1 infected macrophages. Cell viability was measured using FACS analysis after different time points $(0,2,4$, and 6 days) post-co-culture of engineered SK-N-SH cells with HIV-1 infected macrophages (upper panel). SK-N-SH cells stably expressing PAR2 show high mortality rate, but PAR2-Furin expressing cells are significantly protected from PAR2-induced neurotoxicity. $N=3$ (macrophage from three different donors); Bars represent mean $\pm \mathrm{SD}$, and $p$ was determined using one-tailed student $T$-test. Values of $p<$ 0.05 are considered significant and are shown for each condition

in the regulation of the retrograde trafficking of PAR1 to the TGN, even in the absence of Furin (Figs 2B, 3). Deletion of the phosphorylatable cytosolic tail of PAR1 and the expression of this PAR1- $1390-406$ deletant with PACS1 also revealed that this $\mathrm{C}$-terminal PAR1 domain represents a likely binding site to PACS1 (Fig. 3B). Furthermore, siRNA silencing of PACS1 prevents the subcellular entrapment of PAR1 in the TGN (Fig. 3C). Although we provided evidence that the interacting sequence with PACS1 is at the extreme C-terminus of PAR1, the exact motif within such an acidic Ser-rich segment, reported to be phosphorylated [49], has not yet been identified. A plausible acidic motif is ESSDPSS $_{396}$ in PAR1, which resembles the acidic phosphorylated $\operatorname{EEXP}(\mathrm{pS}) \mathrm{D}\left(\mathrm{pS}_{775}\right)$ one in the cytosolic tail of Furin that binds PACS-1 [29].

The highly conserved $\mathrm{Asn}_{47}$ plays an important role in PAR1's ability to interact with Furin. Our data show that PAR1-N47D when expressed with Furin, is no longer trapped in the TGN but is instead localized to the cellsurface (Fig. 4A). Thus, we hypothesize that the N47D mutant interaction with PAR1 is weak at the acidic $\mathrm{pH}$ of the TGN (pH 6.2-6.4) and that most of the receptor is sorted to the cell-surface where it can still inhibit Furin activity (Fig. 4B). It is important to note that proGDF11 is cleaved by PC5 at RSRR ${ }_{296} \downarrow \mathbf{N}$, where the PC-recognition motif in
proGDF11 is followed by Asn. It was reported that when the highly conserved $\mathrm{Asn}_{297}$ is replaced by an Asp, all other basic-amino- acid-specific PCs could cleave proGDF11 as well [32]. Co-incidentally, PAR1 also has an Asn at the P1' position. However, the N47D mutant does not follow this pattern, as it is still cleaved by PC5A and PACE4 but not by Furin, PC5B or PC7 (Supplementary Figure S8), similar to WT PAR1 [10].

We also show that PAR2 N-glycosylation at the P7 position from the cleavage site $\left(\mathrm{NR}_{31} \mathrm{SSKGR}_{36} \downarrow\right)$ affects its cleavage efficacy by Furin and PC7. It was hypothesized that this post-translational modification at $\mathrm{Asn}_{30}$ could result in a steric hindrance [39], and thus may explain why the other PCs are not able to cleave PAR2. Indeed, the natural N30S mutant of PAR2 (rs616235) is not only cleaved by Furin with a better efficiency but also to a lesser extent $(\sim 30 \%)$ by PC7. However, it is still not cleaved by PC5A or PACE4 (Fig. 6B, C).

Thus, PAR1 and PAR2 are so far the only seven transmembrane domain containing receptors identified as inhibitor and substrate of Furin, respectively. The present study presents early events in HAND, where upregulated PCs in the CNS cleave and disarm the inflammatory receptors PAR1,2, thus protecting neurons from inflammatory signaling and cell death. Finally, our results provide novel insights into the PC-PAR1,2 interaction, thereby enhancing our understanding of the early onset and possibly progression of neuroinflammation and neurodegeneration in HAND.

\section{Materials and methods}

\section{Plasmids and reagents}

PAR1 and PAR2 cDNAs were cloned in pcDNA3.1. While PAR1 contains a mcherry and eYFP tag [10], PAR2 contains a mRFP and eYFP tag on its N- and C-termini [34], respectively. Furin, PC5A, PC5B, PACE4, and PC7 were cloned in pIRES2-EGFP vector and tagged with V5 [37]. $\mathrm{HxBc} 2$ Env encoding plasmid was used as described earlier [50]. A pCDNA3.1 plasmid encoding an unrelated protein 7B2 [51] was used as control. Site-directed mutagenesis was used to generate mutants for PAR1 and PAR2 to study their structure-function relationship. Mutants and the primers used are listed in Supplementary Table S1.

\section{Cell culture and transfections}

HEK293 (human embryonic kidney-derived epithelial) cells were grown in DMEM media (Wisent bioproducts) with $10 \%$ FBS and CHO-FD11 cells (Furin-deficient Chinese hamster ovary cells) [37] were grown in DMEM-F12 media 
with 5\% FBS. SK-N-SH (human neuroblastoma) cells were grown in EMEM media (Wisent bioproducts) with $10 \%$ FBS. SK-N-SH cells were differentiated for three days into neurons with $1 \mathrm{mM}$ dibutyryl cAMP (Sigma-Aldrich), as previously described [23]. Transfections for western blot analysis was done using a Jetprime (Polyplus) reagent and Fugene HD (Promega) for Immunofluorescence experiments. Cells were transiently transfected at $60-70 \%$ confluency according to manufacturer's protocol. After $24 \mathrm{~h}$, the cells were incubated in serum-free media for an additional $24 \mathrm{~h}$, whereupon cell lysates were prepared. PAR/PC stable cell lines were prepared following electroporation of the cDNAs into SK-N-SH cells transfected according to Nucleofector transfection reagent protocol (Lonza), followed by G418 antibiotic selection for 14 days. The clones were then sorted by Fluorescence Activated Cell Sorting (FACS) and a stable pool generated of each genotype. Human PACS1 and scramble siRNA were purchased from GE Healthcare Biosciences (Dharmacon; siGENOME SMARTpool; Table S1) and transfected using DhamaFECT 1 transfection reagent (Dharmacon), as recommended by the manufacturer's protocol.

\section{Immunohistochemistry and Immunofluorescence}

Immunohistochemistry of GFAP, PAR1, and PAR2 on brain sections of control and HAND patients were performed as previously reported using the same human antibodies [8, 10, 15]. HEK293 cells or the Furin-deficient CHO-FD11 cells were plated on poly-lysine coated glass coverslips and transfected with appropriate cDNA vectors. After $24 \mathrm{~h}$, the cells were incubated in serum-free media for $20 \mathrm{~h}$, following which the cells were washed with PBS and fixed with 4\% paraformaldehyde for 15 min. The coverslips were then again washed with PBS and mounted on slides with Prolong Gold Antifade reagent with DAPI (Invitrogen). Immunofluorescence analysis was done on a Leica SP8 confocal microscope. Inhibitors of PCs (cell-permeable decanoyl-RVKR-cmk, Bachem Biosciences and non-cell-permeable D6R, EMD chemicals were used at concentrations of $25 \mathrm{nM}$ and $10 \mu \mathrm{M}$, respectively) were added along with the serum-free media $24 \mathrm{~h}$ after transfection and trypsin or anti-trypsin (SigmaAldrich) were added $45 \mathrm{~min}$ before slide preparation. When antibodies were used, the cells were permeabilized with $0.25 \%$ Triton $\mathrm{X}-100$ for $7 \mathrm{~min}$ followed by fixation. Blocking was performed after three washes with PBS using $1 \%$ bovine serum albumin for $30 \mathrm{~min}$. The cells were then incubated with antibodies at 1:500 dilution in 1\% BSA: mouse mAb anti-Golgin97 (Santa Cruz), mouse $\mathrm{mAb}$ anti-V5 (Sigma-Aldrich) or goat polyclonal antiLDLR (R\&D systems) according to the experimental design. Alexa conjugated secondary antibodies
(Invitrogen) were diluted in $1 \%$ bovine serum albumin, and then incubated for $1 \mathrm{~h}$ at room temperature after 3 washes with PBS.

\section{Western blot analysis}

Proteins were extracted in $50 \mathrm{mM}$ Tris- $\mathrm{HCl} \mathrm{pH} 8,150 \mathrm{mM}$ $\mathrm{NaCl}, 0.1 \%$ SDS, $1 \%$ Nonidet $\mathrm{P} 40$ and $0.25 \% \mathrm{Na}$ deoxycholate (RIPA 1X) buffer with a cocktail of protease inhibitors (Roche). Bradford assay was used to evaluate the protein concentrations. Proteins were resolved on SDSPAGE and blotted onto nitrocellulose or PVDF membranes. After incubating the membranes with appropriate primary and secondary antibodies the analysis and quantifications were done using ChemiDoc imaging system (Biorad). Antibodies used: Chessie 8 anti-gp41 [52], rabbit polyclonal anti $\beta$-actin (1:5000; Sigma-Aldrich), rabbit polyclonal antiPACS1 (1:1000; Sigma-Aldrich), mouse mAb-V5 (1:5000; Sigma-Aldrich), mouse mAb eRFP (1:5000; Origene), and appropriate HRP conjugated secondary antibodies. Enzymatic chemiluminescence (Bio-rad) was used to detect immunoreactive species.

\section{Monocyte-derived macrophages co-culture}

Monocyte-derived macrophages (MDM) from Donors at the IRCM were utilized in the co-culture experiments. Macrophages were infected with HIV-1 NL4.3-ADA strain, used at $\mathrm{MOI}=1$. Monocyte-derived macrophages (MDMs) were derived from monocytes isolated from PBMCs obtained by Ficoll Gradients of Blood. These infected macrophages were then co-cultured with PC and/or PAR1/2 stably expressing SK-N-SH cells for 6 days. For co-culture, SK-NSH cells and the stable cell lines were plated in a 24 well plate $(10,000$ cells/well). After $24 \mathrm{~h}$, the media was supplemented with $1 \mathrm{mM}$ dibutyryl cAMP for three days. After $72 \mathrm{~h}$, the isolated macrophages were placed in a 24-well plate cell culture hanging insert (Millipore). These inserts were then placed in the desired well. The viability of these cells was then measured at different time points using the LIVE/DEAD ${ }^{\mathrm{TM}}$ Fixable Yellow Dead Cell Stain Kit (ThermoFisher) and FACS analysis.

\section{Cell viability assay}

The CellTiter 96 AQueous One Solution Cell Proliferation Assay (MTS) was used (Promega). SK-N-SH cells and the stable clones were plated on a 96-well plate. After differentiation, recombinant thrombin $(10 \mathrm{nM})$ was added. At appropriate time points, $20 \mu \mathrm{l}$ of CellTiter $96^{\circledR}$ AQueous One Solution Reagent were added to each well of the 96well assay plate containing the samples in $100 \mu \mathrm{l}$ of culture medium. After incubating the plate at $37^{\circ} \mathrm{C}$ for $2 \mathrm{~h}$ in a 
humidified, $5 \% \mathrm{CO}_{2}$ atmosphere, viability was analyzed by recording the absorbance at $490 \mathrm{~nm}$ using a 96-well plate reader.

Acknowledgements This work was supported by a Canadian Institutes of Health Research Emerging Team Grant on HAND \# TCO125271 (N.G.S., C.P., E.A.C.) and in part by a CIHR Foundation grant \# 148363 (N.G.S). This work was also supported by Canada Research Chairs in Precursor Proteolysis (N.G.S.; \# 950-231335), Neurological Infection and Immunity (C.P.) and Human Retrovirology (E.A.C.). The authors thank Brigitte Mary for editorial help.

\section{Compliance with ethical standards}

Conflict of interest The authors declare that they have no conflict of interest.

Publisher's note: Springer Nature remains neutral with regard to jurisdictional claims in published maps and institutional affiliations.

\section{References}

1. Eggers C, Arendt G, Hahn K, Husstedt IW, Maschke M, NeuenJacob E, et al. HIV-1-associated neurocognitive disorder: epidemiology, pathogenesis, diagnosis, and treatment. J Neurol. 2017;264:1715-27.

2. Mcarthur JC, Hoover DR, Bacellar H, Miller EN, Cohen BA, Becker JT, et al. Dementia in AIDS patients: incidence and risk factors. Multicenter AIDS Cohort Study. Neurology. 1993;43:2245-52.

3. Woods SP, Moore DJ, Weber E, Grant I. Cognitive neuropsychology of HIV-associated neurocognitive disorders. Neuropsychol Rev. 2009;19:152-68.

4. Antinori A, Arendt G, Becker JT, Brew BJ, Byrd DA, Cherner M, et al. Updated research nosology for HIV-associated neurocognitive disorders. Neurology. 2007;69:1789-99.

5. Perry S, Marotta RF. AIDS dementia: a review of the literature. Alzheimer Dis Assoc Disord. 1987;1:221-35.

6. Gelman BB. Neuropathology of HAND with suppressive antiretroviral therapy: encephalitis and neurodegeneration reconsidered. Curr HIV/AIDS Rep. 2015;12:272-9.

7. Noorbakhsh F, Vergnolle N, Mcarthur JC, Silva C, Vodjgani M, Andrade-Gordon $\mathrm{P}$, et al. Proteinase-activated receptor-2 induction by neuroinflammation prevents neuronal death during HIV infection. J Immunol. 2005;174:7320-9.

8. Boven LA, Vergnolle N, Henry SD, Silva C, Imai Y, Holden J, et al. Upregulation of proteinase-activated receptor 1 expression in astrocytes during HIV encephalitis. J Immunol. 2003;170:263846.

9. Seidah NG, Prat A. The biology and therapeutic targeting of the proprotein convertases. Nat Rev Drug Discov. 2012; 11:367-83.

10. Kim W, Zekas E, Lodge R, Susan-Resiga D, Marcinkiewicz E, Essalmani R, et al. Neuroinflammation-induced interactions between protease-activated receptor 1 and proprotein convertases in HIV-associated neurocognitive disorder. Mol Cell Biol. 2015;35:3684-700.

11. Adams MN, Ramachandran R, Yau MK, Suen JY, Fairlie DP, Hollenberg MD, et al. Structure, function and pathophysiology of protease activated receptors. Pharmacol Ther. 2011;130:248-82.

12. Soh UJ, Dores MR, Chen B, Trejo J. Signal transduction by protease-activated receptors. Br J Pharmacol. 2010;160:191-203.
13. Ramachandran R, Noorbakhsh F, Defea K, Hollenberg MD. Targeting proteinase-activated receptors: therapeutic potential and challenges. Nat Rev Drug Discov. 2012;11:69-86.

14. Steinhoff M, Buddenkotte J, Shpacovitch V, Rattenholl A, Moormann C, Vergnolle N, et al. Proteinase-activated receptors: transducers of proteinase-mediated signaling in inflammation and immune response. Endocr Rev. 2005;26:1-43.

15. Noorbakhsh F, Tsutsui S, Vergnolle N, Boven LA, Shariat N, Vodjgani M, et al. Proteinase-activated receptor 2 modulates neuroinflammation in experimental autoimmune encephalomyelitis and multiple sclerosis. J Exp Med. 2006;203:425-35.

16. Lee PR, Johnson TP, Gnanapavan S, Giovannoni G, Wang T, Steiner JP, et al. Protease-activated receptor-1 activation by granzyme B causes neurotoxicity that is augmented by interleukin-1beta. J Neuroinflamm. 2017;14:131.

17. Moller T, Hanisch UK, Ransom BR. Thrombin-induced activation of cultured rodent microglia. J Neurochem. 2000;75:1539-47.

18. Smith-Swintosky VL, Cheo-Isaacs CT, D'andrea MR, Santulli RJ, Darrow AL, Andrade-Gordon P. Protease-activated receptor-2 (PAR-2) is present in the rat hippocampus and is associated with neurodegeneration. J Neurochem. 1997;69:1890-6.

19. Seidah NG, Chretien M. Proprotein and prohormone convertases: a family of subtilases generating diverse bioactive polypeptides. Brain Res. 1999;848:45-62.

20. Okamoto M, Wang X, Debyser Z, De Clercq E, Baba M. Establishment of an in vitro assay system mimicking human immunodeficiency virus type 1-induced neural cell death and evaluation of inhibitors thereof. J Virol Methods. 2003;108:195-203.

21. Rodriguez-Franco EJ, Cantres-Rosario YM, Plaud-Valentin M, Romeu R, Rodriguez Y, Skolasky R, et al. Dysregulation of macrophage-secreted cathepsin B contributes to HIV-1-linked neuronal apoptosis. PLoS ONE. 2012;7:e36571.

22. Li W, Gorantla S, Gendelman HE, Poluektova LY. Systemic HIV1 infection produces a unique glial footprint in humanized mouse brains. Dis Model Mech. 2017;10:1489-502.

23. Maingat F, Viappiani S, Zhu Y, Vivithanaporn P, Ellestad KK, Holden J, et al. Regulation of lentivirus neurovirulence by lipopolysaccharide conditioning: suppression of CXCL10 in the brain by IL-10. J Immunol. 2010;184:1566-74.

24. Moriuchi H, Moriuchi M, Fauci AS. Cathepsin G, a neutrophilderived serine protease, increases susceptibility of macrophages to acute human immunodeficiency virus type 1 infection. J Virol. 2000;74:6849-55.

25. Dubois CM, Blanchette F, Laprise MH, Leduc R, Grondin F, Seidah NG. Evidence that furin is an authentic transforming growth factor-beta1- converting enzyme. Am J Pathol. 2001;158:305-16.

26. Dobolyi A, Vincze C, Pal G, Lovas G. The neuroprotective functions of transforming growth factor beta proteins. Int $\mathrm{J}$ Mol Sci. 2012;13:8219-58.

27. Wan L, Molloy SS, Thomas L, Liu G, Xiang Y, Rybak SL, et al. PACS-1 defines a novel gene family of cytosolic sorting proteins required for trans-Golgi network localization. Cell. 1998; 94:205-16.

28. Crump CM, Xiang Y, Thomas L, Gu F, Austin C, Tooze SA, et al. PACS-1 binding to adaptors is required for acidic cluster motifmediated protein traffic. EMBO J. 2001;20:2191-201.

29. Thomas G. Furin at the cutting edge: from protein traffic to embryogenesis and disease. Nat Rev Mol Cell Biol. 2002;3:75366.

30. Shapiro MJ, Trejo J, Zeng D, Coughlin SR. Role of the thrombin receptor's cytoplasmic tail in intracellular trafficking. Distinct determinants for agonist-triggered versus tonic internalization and intracellular localization. J Biol Chem. 1996;271:32874-80. 
31. Gordon VM, Rehemtulla A, Leppla SH. A role for PACE4 in the proteolytic activation of anthrax toxin protective antigen. Infect Immun. 1997;65:3370-5.

32. Essalmani R, Zaid A, Marcinkiewicz J, Chamberland A, Pasquato A, Seidah NG, et al. In vivo functions of the proprotein convertase PC5/6 during mouse development: Gdf11 is a likely substrate. Proc Natl Acad Sci USA. 2008;105:5750-5.

33. Gelman BB, Endsley J, Kolson D. When do models of NeuroAIDS faithfully imitate "the real thing. J Neurovirol. 2018;24: 146-55.

34. Mihara K, Ramachandran R, Saifeddine M, Hansen KK, Renaux B, Polley D, et al. Thrombin-mediated direct activation of proteinase-activated receptor-2: another target for thrombin signaling. Mol Pharmacol. 2016;89:606-14.

35. Cottrell GS, Amadesi S, Schmidlin F, Bunnett N. Proteaseactivated receptor 2: activation, signalling and function. Biochem Soc Trans. 2003;31:1191-7.

36. Miike S, Mcwilliam AS, Kita H. Trypsin induces activation and inflammatory mediator release from human eosinophils through protease-activated receptor-2. J Immunol. 2001;167:6615-22.

37. Susan-Resiga D, Essalmani R, Hamelin J, Asselin MC, Benjannet $\mathrm{S}$, Chamberland A, et al. Furin is the major processing enzyme of the cardiac-specific growth factor bone morphogenetic protein 10 . J Biol Chem. 2011;286:22785-94.

38. Essalmani R, Susan-Resiga D, Guillemot J, Kim W, Sachan V, Awan $\mathrm{Z}$, et al. Thrombin activation of protein $\mathrm{C}$ requires prior processing by a liver proprotein convertase. J Biol Chem. 2017;292:10564-73.

39. Compton SJ, Sandhu S, Wijesuriya SJ, Hollenberg MD. Glycosylation of human proteinase-activated receptor-2 (hPAR2): role in cell surface expression and signalling. Biochem J. 2002;368:495-505.

40. Saylor D, Dickens AM, Sacktor N, Haughey N, Slusher B, Pletnikov $\mathrm{M}$, et al. HIV-associated neurocognitive disorder-pathogenesis and prospects for treatment. Nat Rev Neurol. 2016;12: 234-48.

41. Walsh JG, Reinke SN, Mamik MK, Mckenzie BA, Maingat F, Branton WG, et al. Rapid inflammasome activation in microglia contributes to brain disease in HIV/AIDS. Retrovirology. 2014;11:35.

42. Striggow F, Riek-Burchardt M, Kiesel A, Schmidt W, HenrichNoack P, Breder J, et al. Four different types of protease-activated receptors are widely expressed in the brain and are up-regulated in hippocampus by severe ischemia. Eur J Neurosci. 2001; 14:595-608

43. Noorbakhsh F, Vergnolle N, Hollenberg MD, Power C. Proteinase-activated receptors in the nervous system. Nat Rev Neurosci. 2003;4:981-90.

44. Dihanich M, Kaser M, Reinhard E, Cunningham D, Monard D. Prothrombin mRNA is expressed by cells of the nervous system. Neuron. 1991;6:575-81.

45. Shikamoto $Y$, Morita T. Expression of factor $X$ in both the rat brain and cells of the central nervous system. FEBS Lett. 1999;463:387-9.

46. Reed DE, Barajas-Lopez C, Cottrell G, Velazquez-Rocha S, Dery $\mathrm{O}$, Grady EF, et al. Mast cell tryptase and proteinase-activated receptor 2 induce hyperexcitability of guinea-pig submucosal neurons. J Physiol. 2003;547:531-42.

47. Theoharides TC. Mast cells: the immune gate to the brain. Life Sci. 1990;46:607-17.

48. Cordova ZM, Gronholm A, Kytola V, Taverniti V, Hamalainen S, Aittomaki S, et al. Myeloid cell expressed proprotein convertase FURIN attenuates inflammation. Oncotarget. 2016;7:54392-404.

49. Hammes SR, Shapiro MJ, Coughlin SR. Shutoff and agonisttriggered internalization of protease-activated receptor 1 can be separated by mutation of putative phosphorylation sites in the cytoplasmic tail. Biochemistry. 1999;38:9308-16.

50. Lodge R, Lalonde JP, Lemay G, Cohen EA. The membraneproximal intracytoplasmic tyrosine residue of HIV-1 envelope glycoprotein is critical for basolateral targeting of viral budding in MDCK cells. EMBO J. 1997;16:695-705.

51. Seidah NG, Hsi KL, De Serres G, Rochemont J, Hamelin J, Antakly $\mathrm{T}$, et al. Isolation and $\mathrm{NH} 2$-terminal sequence of a highly conserved human and porcine pituitary protein belonging to a new superfamily. Immunocytochemical localization in pars distalis and pars nervosa of the pituitary and in the supraoptic nucleus of the hypothalamus. Arch Biochem Biophys. 1983; 225:525-34.

52. Abacioglu YH, Fouts TR, Laman JD, Claassen E, Pincus SH, Moore JP, et al. Epitope mapping and topology of baculovirusexpressed HIV-1 gp160 determined with a panel of murine monoclonal antibodies. AIDS Res Hum Retrovir. 1994; $10: 371-81$. 